\title{
Mathematical Simulation of Defecate Distribution by a Centrifugal Working Tool
}

\author{
Nikolay Petrovich Kolesnikov \\ Candidate of Engineering Sciences, Associate Professor of \\ the Department of Transport and Technological Machine \\ Operation \\ Voronezh State Agrarian University named after Emperor \\ Peter the Great \\ Voronezh, Russia \\ email: nikolay2060@yandex.ru \\ Vladimir Pavlovich Shatskiy \\ Doctor of Engineering Sciences, Professor of the \\ Department of Mathematics and Physics \\ Voronezh State Agrarian University named after Emperor \\ Peter the Great \\ Voronezh, Russia \\ e-mail: mathem@agroeng.vsau.ru
}

\author{
Anatoliy Petrovich Dyachkov \\ Candidate of Engineering Sciences, Professor of the \\ Department of Transport and Technological Machine \\ Operation \\ Voronezh State Agrarian University named after Emperor \\ Peter the Great \\ Voronezh, Russia \\ e-mail: kafexpl@agroeng.vsau.ru \\ Ivan Alekseevich Spitsyn \\ Doctor of Engineering Sciences, Professor of the \\ Department of Fundamentals of Mechanism and Machine \\ Design, Penza State Agrarian University, \\ Penza Russia \\ e-mail:spicn@mail.ru
}

\author{
Aleksey Dmitrievich Brovchenko \\ Candidate of Engineering Sciences, Associate Professor of the \\ Department of Transport and Technological Machine Operation \\ Voronezh State Agrarian University named after Emperor Peter the Great \\ Voronezh, Russia \\ e-mail: broaldot@yandex.ru
}

\begin{abstract}
The article is devoted to theoretical studies of defecate distribution over the soil surface by a centrifugal working tool. Defecate has physical and mechanical properties which are similar to those of mineral fertilizers. Therefore, for its distribution, fertilizer spreaders with centrifugal working elements are used. However, without structural changes, their use is limited due to increasing defecate weight fed to the working tool $(12-60 \mathrm{~kg} / \mathrm{s})$. It is necessary to determine parameters of the working tool for defecate distribution. To solve this problem, a method for calculating defecate distribution over the soil surface has been developed. The method is implemented in the form of a computer program used to study the nature of defecate distribution depending on the following variables: defecate particle size; a blade installation angle on the disk surface; blade lengths; disk conicity; disc installation height above the soil surface; disc rotation speed; distance between disks (for a two-disc spreader) and feed zone parameters (location, shape, material entry unevenness).
\end{abstract}

Keywords - Defecate; centrifugal working tool; mathematical simulation.

\section{INTRODUCTION}

Physical phenomena occurring in the flow of particles of the spread material with different particle sizes and aerodynamic characteristics are understudied. This is due to the complex nature of interaction of individual particles of irregular shape, a working tool and the air medium which is difficult to describe using mathematical methods $[1,2]$.

However, the nature of interaction can be identified by adopting some assumptions when considering conditions for interaction of particles, working tool and the environment.

The workflow of a centrifugal device involves several phases: material feed to the centrifugal disk; movement of particles along the blades; free flight of particles in the air followed by their contact with the soil surface; landing of particles and their distribution over the field surface $[1,3]$.

All these phases are closely interrelated, moreover, in each of them there are a large number of factors that directly or indirectly influence the nature of material distribution over the soil surface. It is very difficult to take into account the influence of these factors. Therefore, researchers are forced to make assumptions. 
Analysis of previous theoretical studies [4-12] identified that the authors make the following assumptions:

- when feeding fertilizers to the disk through the tube line, all particles fall into one point corresponding to the center of the tube line;

- with a sufficiently extensive fertilizer feed to the disc, calculations are carried out for the extreme points of the feed zone;

- averaged values characterizing physicomechanical and granulometric properties of the spread material are taken into account (it is assumed that all particles are homogeneous).

The assumptions affect calculation accuracy. They can be used for materials which are equal by their granulometric composition and physicomechanical properties. The material is fed to the disk through a small diameter pipeline. This is possible only when small doses of fertilizers are introduced into the soil. For defecate which differs in various granulometric composition and is introduced in large doses, it is hardly applicable.

We attempted to simulate the defecate distribution process involving material feed to the working tool of the spreader and material spreading over the soil surface.

\section{METHOD}

The zone of the material feed to the working tool is broken by a grid along the axes of the coordinate system. It is beleived that particles of different weight fall into each grid node in proportions corresponding to the particle size distribution of the material. By varying uniformity of the grid, one can take into account the uneven flow of particles in the feed zone. Thus, it creates an array of possible options for particles to fall into the working tool.

To study forces acting on a particle which fell on a centrifugal working tool and determine its relative movement speed, let us introduce a moving coordinate system XOY, rigidly connected with the disk, in a plane passing through the base of the blade and parallel disc radius starting in its center. The axis of rotation is denoted by $\mathrm{Z}$, and the rotation itself is counterclockwise (Fig. 1). 15]:

The following forces act on a particle fallen on a disk [13-

1. Gravity force mg. The projection of this force on the $X$ axis will be equal to $\mathrm{mgcos} \gamma$. This force creates the force of friction of the particle on the disc surface of the disk.

2. Centrifugal force $m r \omega^{2}$ acting in the radial direction. If the blade is located at angle $\alpha_{\ell}$ to the radial direction, the centrifugal force can be divided into two components. The component acting in the blade direction is equal $\mathrm{mx} \omega^{2} \cos \gamma$. The component which is perpendicular to the blade plane is $m \omega^{2} L_{y} \operatorname{tg} \alpha_{\ell} \cos \gamma$. These components participate in creation of the friction force acting in the blade direction from its edge to the disc center. Component $\mathrm{mx} \omega^{2} \cos \gamma$ gravity force projection create the force of friction the disc surface.

$$
\mathrm{F}_{\mathrm{Tp} 2}=\mathrm{fN}_{2}=\mathrm{f}\left(\mathrm{mg} \cos \gamma+\mathrm{m} \omega^{2} \cos \gamma \cdot \mathrm{x} \cdot \sin \gamma\right)
$$

where $\mathrm{f}$ - coefficient of material friction on the disc surface; $\mathrm{N}_{2}$ - normal response of the disc surface, $\mathrm{N}$;

$\gamma$ - disc conicity, rad; $\omega-$ angular disc rotation speed, $\operatorname{rad}^{-1}$, $\mathrm{x}$ - current point coordinate $\mathrm{x}, \mathrm{m}$.

3. The Coriolis force- $2 m \omega \dot{x} \cos \gamma$ where $\dot{x}$ - speed of the particle movement, $\mathrm{m} / \mathrm{s}$. The direction of this force is perpendicular to the direction of relative speed and is oriented in the direction which is opposite to the rotation direction. This force and the centrifugal force cause the force of normal blade N1 response which creates friction of the particle on the blade surface which is equal to

$$
\mathrm{F}_{\mathrm{Tpl}}=\mathrm{fN}_{1}=\mathrm{f}\left(2 \mathrm{~m} \omega \cdot \dot{\mathrm{x}} \cdot \cos \gamma+\mathrm{m} \omega^{2} \mathrm{~L}_{\mathrm{y}} \operatorname{tg} \alpha_{\ell} \cos \gamma\right),
$$

where $\mathrm{L}_{\mathrm{y}}$ - blade length, $\mathrm{m}$.

The friction coefficient $\mathrm{f}$ is assumed to be the same for blade and disc surfaces.

4. Inertia force $\mathrm{P}=\mathrm{m} \ddot{x}$, where $\ddot{x}$ is acceleration of the particle moving along the blade of the working tool, $\mathrm{m} / \mathrm{s}^{2}$.
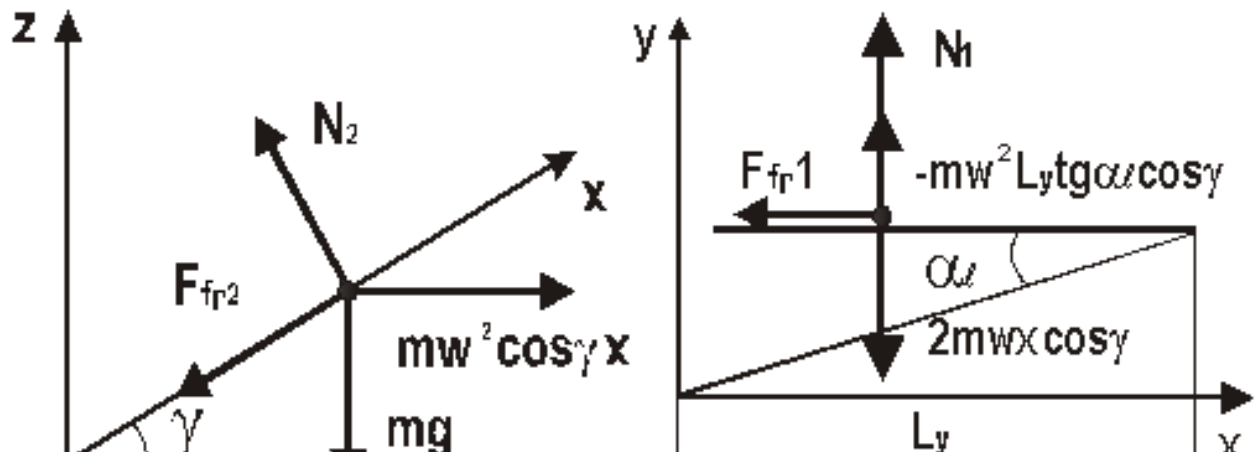

Fig. 1. Forces acting on the particle moving along the blade 
Let us project all the forces acting on the particle on the $\mathrm{X}$ axis. According to the equilibrium condition, the sum of projections of all forces is euqal to zero.

$$
\mathrm{mg} \sin \gamma-\mathrm{m} \omega^{2} \cos ^{2} \gamma \cdot \mathrm{r} \cdot \cos \alpha_{\ell}+\mathrm{F}_{\mathrm{rp} 1}+\mathrm{F}_{\mathrm{rp} 2}+\mathrm{P}=0
$$

where $r$ - current point radius, $m$.

Assume that $r \cos \alpha_{\ell}=\mathrm{x}$ equation of particle movement along the blade can be written as

$$
m \ddot{x}=m \omega^{2} \cos ^{2} \gamma \cdot x-F_{\text {тр2 }}-m g \sin \gamma-F_{\text {тp1 }} .
$$

Having inserted equations ( 1 and 2) in equation (4), we will have a differential equation describing particle movement in the blade direction

$$
\begin{aligned}
& \quad \mathrm{m} \ddot{x}=\mathrm{m} \omega^{2} \cos ^{2} \gamma \cdot x-f\left(\mathrm{mg} \cos \gamma+\mathrm{m} \omega^{2} \cos \gamma \cdot x \cdot \sin \gamma\right)- \\
& -\mathrm{mg} \sin \gamma-\mathrm{f}\left(2 \mathrm{~m} \omega \dot{\mathrm{x}} \cos \gamma+\mathrm{m} \omega^{2} \mathrm{~L}_{\mathrm{y}} \operatorname{tg} \alpha \ell \cos \gamma\right) .
\end{aligned}
$$

This equation includes such variable values as conicity, a blade installation angle, a disk angular velocity, a blade length (disk diameter), a friction coefficient.

Having transformed the equation and reduced by mass $m$, we have

$$
\begin{aligned}
& \ddot{x}=\omega^{2} \cos \gamma(\cos \gamma-f \sin \gamma) x-2 f \omega \dot{x} \cos \gamma- \\
& -g(\sin \gamma+f \cos \gamma)-f \omega^{2} L_{y} \operatorname{tg} \alpha_{\ell} \cos \gamma
\end{aligned}
$$

Assuming the relative initial velocity of the point along the $\mathrm{X}$ axis to be zero, which is typical of the vertical material feed, we can have a standard solution of equation (6)

$$
\mathrm{x}(\mathrm{t})=\mathrm{C}_{1} \mathrm{e}^{\Omega_{1} \mathrm{t}}-\mathrm{C}_{2} \mathrm{e}^{\Omega_{2} \mathrm{t}}+\frac{\mathrm{g}(\sin \gamma+\mathrm{f} \cdot \cos \gamma)+\mathrm{f} \omega^{2} \mathrm{~L} \mathrm{tg} \alpha \cdot \cos \gamma}{\omega^{2} \cos \gamma(\cos \gamma-\mathrm{f} \sin \gamma)}
$$

$$
\mathrm{V}_{\text {iòi }}=\mathrm{C}_{1} \Omega_{1} \mathrm{e}^{\Omega_{1 \mathrm{t}}}-\mathrm{C}_{2} \Omega_{2} \mathrm{e}^{\Omega_{2} \mathrm{t}} \text {, }
$$

where $\mathrm{C}_{1}, \mathrm{C}_{2}$ - arbitrary constants; $\Omega_{1}, \Omega_{2}$ - rots of the characteristic equation.

$$
\Omega 1,2=-\mathrm{f} \omega \cos \gamma \pm \omega \sqrt{\mathrm{f}^{2} \cos ^{2} \gamma+\cos \gamma(\cos \gamma-\mathrm{f} \sin \gamma)},
$$

$$
\mathrm{Cl}, 2=\Omega 1,2 \frac{\mathrm{xo}-\frac{\mathrm{g}(\sin \gamma+\mathrm{f} \cos \gamma)+\mathrm{f} \omega^{2} \mathrm{Ly} \operatorname{tg} \alpha \cos \gamma}{\omega^{2} \cos \gamma(\cos \gamma-\mathrm{f} \sin \gamma)}}{2 \omega \sqrt{\mathrm{f}^{2} \cos ^{2} \gamma+\cos \gamma(\cos \gamma-\mathrm{f} \sin \gamma)}},
$$

where $\mathrm{x}_{0}$ - initial relative coordinate of the point, $\mathrm{m}$.

having calculated relative velocity of the particle movement for the blade end, it is possible to determine the particle fall speed taking into account the blade angle

$$
V_{c x}=\sqrt{\left(\frac{\omega L_{y}}{\cos \alpha_{\ell}}\right)^{2}+V_{r e l}{ }^{2}+\frac{2 \omega L_{y} V_{r e l} \cdot \sin \left(\alpha_{\ell}-\alpha_{u}\right)}{\cos \alpha_{\ell}}},
$$

where $\alpha_{\mathrm{H}}-$ angle of material adhesion to the blade end, rad.

To determine distribution of particles over the soil surface, we introduced a fixed $\mathrm{XaOYa}$ coordinate system which is parallel to the field surface starting in the disc center. The axis OYa is directed tangentially to the trajectory of the centrifugal spreade.r The axis OXa is perpendicular to the axis OYa.

To determine absolute point coordinates for particles falling from the disc, we associate them with relative coordinates. Let us study the projection of the relative coordinate system on the absolute one for the moment when particles interact with the disk blade (Fig. 2).

Figure 2 shows that

$$
y_{a}=\frac{y_{r e l}}{\cos \beta}+x \cdot \operatorname{tg} \beta
$$

$$
V_{c x}=\sqrt{\left(\frac{\omega L_{y}}{\cos \alpha_{\ell}}\right)^{2}+V_{r e l}{ }^{2}+\frac{2 \omega L_{y} V_{r e l} \cdot \sin \left(\alpha_{\ell}-\alpha_{u}\right)}{\cos \alpha_{\ell}}},
$$

At $\operatorname{tg} \beta=\tau$ we have

$$
y_{a}=y_{\text {rel }}\left[\tau^{2}+1\right]+x_{a \tau} .
$$

It follows that

$$
\tau=\frac{\left.\mathrm{xa} \cdot \mathrm{ya} \pm \sqrt{\mathrm{xa}^{2} \cdot \mathrm{ya}^{2}-\left(\mathrm{xa}^{2}-\left(-\mathrm{L}_{\mathrm{y}} \operatorname{tg} \alpha\right)^{2}\right) \cdot\left(\mathrm{ya}^{2}-(-\mathrm{Ly} \operatorname{tg} \alpha)^{2}\right)}\right)}{\mathrm{xa}^{2}-\left(-\mathrm{L}_{\mathrm{y}} \operatorname{tg} \alpha_{\ell}\right)^{2}} .
$$

Let us determine the point coordinate in the absolute coordinate system after $\Delta \mathrm{t}$ over which the disc turns through angle $\beta$. Given that $\frac{\mathrm{dx}}{\mathrm{dt}}=\mathrm{x}_{0} \cos \gamma-$ projection of the relative coordinate of the particle on XaOYa, we have

$$
x_{0} \cos \gamma=\frac{x a}{\cos \beta}+y_{\text {оги }} \text { tg } \beta,
$$




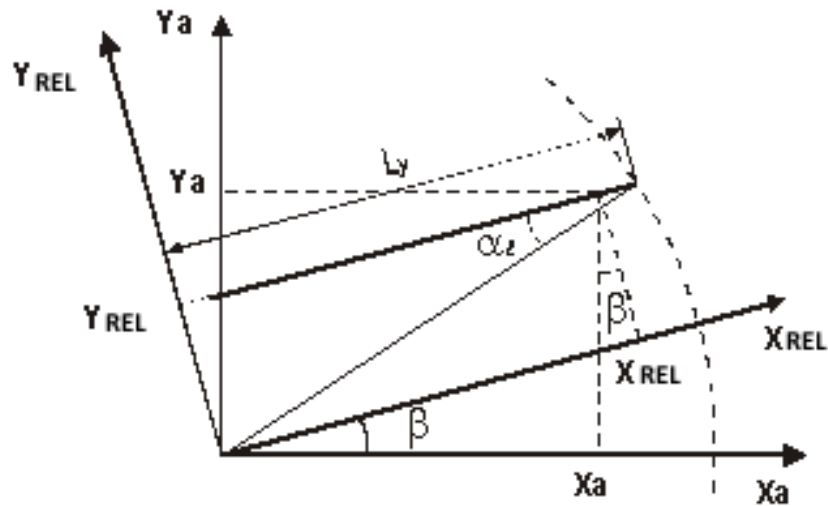

Fig. 2. Connection of absolute and relative coordinates of the particle in the loading zone

or

$$
x_{0} \cos \gamma=\frac{x a}{\cos \beta}+y_{\text {rel }} \operatorname{tg} \beta
$$

Consequently, the point coordinate in the relative coordinate system is equal to

$$
\mathrm{Xo}=\frac{\mathrm{Xa} \sqrt{\tau^{2}+1}-\operatorname{Ly} \operatorname{tg} \alpha \ell \tau}{\cos \gamma}
$$

Thus, absolute coordinates of particles falling on the disk can be determined by their initial relative coordinates $\mathrm{x} 0$ which are sued to determine absolute coordinates of the points of particles falling from the disk.

These parameters can be used to determine the flight distance and coordinates of the particles on the field surface.

To calculate absolute point coordinates for particles falling from the disk, we used Figure 3.

The point of the particle hitting the blade has angle $\Theta$. During the particle movement along the blade $t_{\mathrm{K}}$, the disc turns around angle $\beta$ which is equal to $\beta=\omega t_{\kappa}$.

Thus, denoting the particle falling angle through $\varphi=\beta+\Theta$, we obtain absolute coordinates of the falling point

$$
x_{c o}=L_{y} \cos \phi
$$

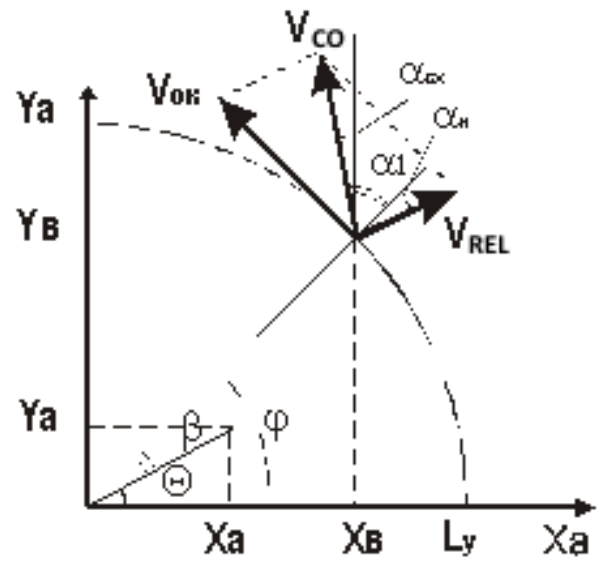

Fig. 3. Fall of particles from the working tool

$$
y_{c o}=L_{y} \sin \phi \text {. }
$$

The angle between the velocity vector and the spreader movement direction can be calculated by formula

$$
\alpha_{c o}=\alpha_{1}+\phi-\frac{\pi}{2}+\alpha_{H}
$$

where $\alpha_{1}-$ angle between the radius and velocity vector Vco, rad.

$$
\alpha_{1}=\arccos \left(\frac{V_{C O}^{2}+V_{R E L}^{2}-V_{O K}^{2}}{2 V_{C O} \cdot V_{R E L}}\right) .
$$

Having determined the absolute coordinates of the falling point, value and direction of the velocity vector of particles falling from the disk, let us consider the flying distance for any particle in the array entering the feed zone.

In this regard, let us consider the motion of the center of mass of a single particle falling from the centrifugal working tool with initial velocity $\dot{x}_{n}(0)=V_{c o} \cos \gamma ; \dot{y}_{n}(0)=V_{c o} \sin \gamma$, at distance $\mathrm{h}$ from the ground surface at an angle $\gamma$ (Fig. 4). 


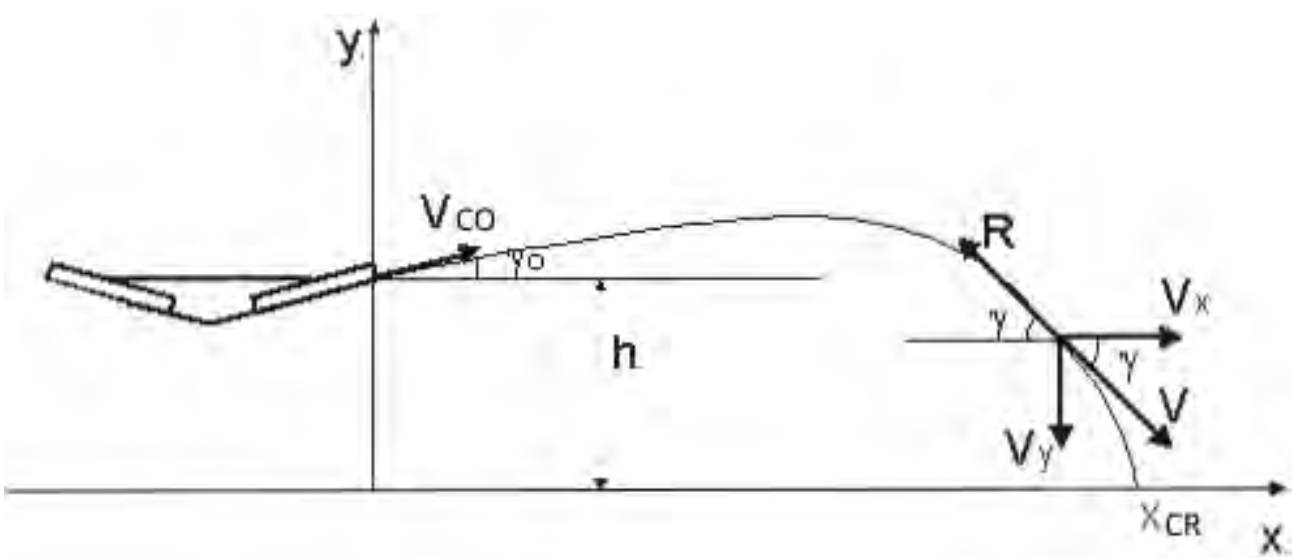

Fig. 4. Scheme for calculation of the flying distance for a particle

A particle is taken as a material point of mass m moving under two forces: weight $\mathrm{mg}$ and air resistance Rair, directed tangentially to the particle trajectory in the direction opposite to the motion and being a given function of velocity $\mathrm{V}$, i.e. $\mathrm{P}_{\text {во3 }}=\mathrm{mf}(\mathrm{V})$.

Air resistance to particle movement at particle velocity varying from 10 to $30 \mathrm{~m} / \mathrm{s}$ is calculated by formula $\mathrm{mf}(\mathrm{V})=\mathrm{mk}_{\mathrm{n}} \mathrm{V}^{2}[14,15]$.

Differential particle flight equations with initial conditions $\mathrm{x}_{\text {п }}(0)=0$ и $\mathrm{y}_{\text {п }}(0)=$ h determined by output parameters of falling particles are standard and can be written as

$$
\begin{gathered}
m \ddot{x}=-m k_{p} V^{2} \frac{\dot{x} n}{\sqrt{\dot{x}_{n}^{2}+\dot{y}_{n}^{2}}}, \\
m \ddot{y}=-m g-m k_{p} \frac{\dot{y} n}{\sqrt{\dot{x}^{2}+\dot{y}^{2}}},
\end{gathered}
$$

Given that $V=\sqrt{\dot{\mathrm{x}}^{2}+\dot{\mathrm{y}}^{2}}$ and having reduced $\mathrm{m}$ in both parts of the equations, the equations can be written as

$$
\begin{gathered}
\ddot{\mathrm{x}}=-\mathrm{kp} \sqrt{\dot{\mathrm{x}}^{2}+\dot{\mathrm{y}}^{2}} \cdot \dot{\mathrm{x}} \mathrm{n}, \\
\ddot{\mathrm{y}}=-\mathrm{g}-\mathrm{kp} \sqrt{\dot{\mathrm{x}}^{2}+\dot{\mathrm{y}}^{2}} \cdot \dot{\mathrm{y}} \mathrm{n}, .
\end{gathered}
$$

This system is not linear, it is solved by numerical methods $[16,17]$. The solution of this system allows us to determine the flight distance for particles and points on the distribution zone surface they fall into.

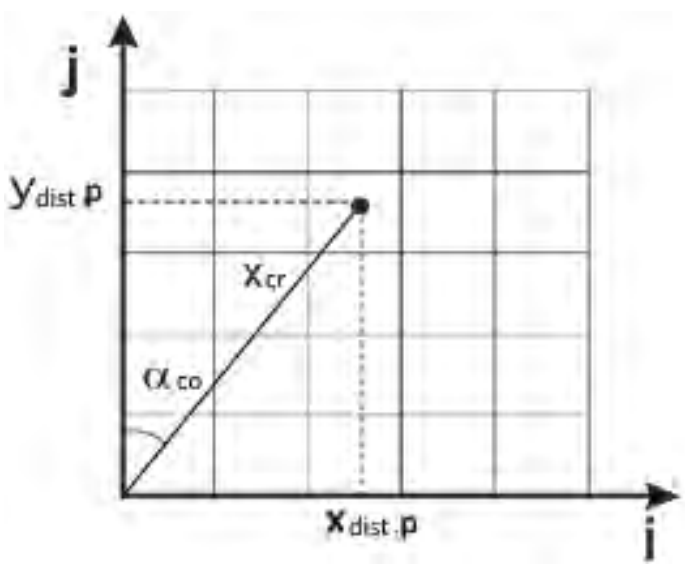

Fig. 5. Scheme of calculation of coordinates of particle distribution along the field surface

Figure 5 shows that the coordinates of particles distribution depend on the flight distance and angle $\alpha_{\mathrm{co}}$.

$$
x_{\mathrm{DIST}, \rho}=x_{\mathrm{CR}} \sin \alpha_{\mathrm{CO}},
$$

$$
x_{\mathrm{DIST}, \rho}=x_{\mathrm{CR}} \cos \alpha_{\mathrm{CO}},
$$

Wherex distrt $, \mathrm{p}, \mathrm{y}_{\text {distr }} \mathrm{p}$ - coordinates $\mathrm{x}$ and $\mathrm{y}$ of distribution of the particle with the $p$-th sailing capacity, $m$; $x_{\mathrm{cr}}, \mathrm{y}_{\mathrm{cr}}-$ flight distance of the particle with the $\mathrm{p}$-th sailing capacity, $\mathrm{m}$.

To determine the distribution of particles across the grip width, we introduced a new coordinate system ioj and accepted new notation.

$$
\begin{aligned}
& \mathrm{i}_{\mathrm{p}}=\left[\mathrm{x}_{\text {dist }} \mathrm{p}\right] \\
& \mathrm{j}_{\mathrm{p}}=\left[\mathrm{y}_{\text {dist }} \mathrm{p}\right]
\end{aligned}
$$

Coordinates of distribution and the number of particles of different sail sizes falling into one or another square of the absolute coordinate system (distribution zone) can be used to determine the percentage distribution of particles of different 
fractional composition across the grip width using the following expression

$$
\mathrm{P}[i-\mathrm{RMC}]=\frac{100}{N i} \sum_{p=1}^{p_{n}} \sum_{j=0}^{\mathrm{m}} R p, i, j,
$$

where $\mathrm{RMC}$ is the distance between the disc centers, $\mathrm{m}$; $\mathrm{Ni}$ is the number of particles trapped in the i-th strip of the track width; $p$ is the fraction number of the spread material; $i, j$ are the numbers of bands, respectively, along the width and length of the track; Rp, i, j is the number of particles of the $p$-th fraction fallen into rectangle $i$ across the track width and $j$ along the track length (with coordinates $\mathrm{i}-\mathrm{j}$ ).

\section{CONCLUSION}

The mathematical method was implemented in the form of a POLET program which helps determine one-to-one correspondence between feed and distribution zones. This fact makes it possible to investigate the nature of the distribution zone depending on the following variables: particle size; blade installation angle on the disk surface; blade lengths; disk conicity; disc installation height above the soil surface; disc rotation speed; distances between disks (for a two-disc spreader); feed zone parameters (location, shape, material entry unevenness).

By varying these parameters, it is possible to determine their influence on the nature of material distribution to reduce unevenness.

\section{References}

[1] P. Van Liedekerke, E. Tijskens, E. Dintwa, F. Rioual and H. Ramon "DEM simulations of the particle flow on a centrifugal fertilizer spreader," Powder Technology, Vol. 190, No. 3, 25 March 2009, pp. 348360

[2] P. Van Liedekerke, E. Tijskens, E. Dintwa, J. Anthonis and H. Ramon "A discrete element model for simulation of a spinning disc fertilizer spreader I. Single particle simulations," Powder Technology, Vol. 170, No. 2, December 2006, pp. 71-85.

[3] A. Przywara, "The Impact of Structural and Operational Parameters of the Centrifugal Disc spreader on the Spatial Distribution of Fertilizer," Agriculture and Agricultural Science Procedia, Vol. 7, 2015, pp. 215-222.
[4] J. W. Hofstee and W. Huisman, "Handling and spreading of fertilizers: Part 1, Physical properties of fertilizer in relation to particle motion," Journal of Agricultural Engineering Research, Vol. 53, SeptemberDecember 1992, pp. 141-162.

[5] J. W. Hofstee "Handling and spreading of fertilizers: Part 2, Physical properties of fertilizer, measuring methods and data," Journal of Agricultural Engineering Research, Vol. 53, September-December 1992, pp. 141-162.

[6] J. M. Petpersen, J. A. Svendsen and S. Øvland "A method of studying the influence of fertilizer particle size on the distribution from a twin-disc spreader," Journal of Agricultural Engineering Research, Vol. 50, September-December 1991, pp. 291-303.

[7] E. -M. Abbou-ou-cherif, E. Piron, A. Chateauneuf, D. Miclet, R.Lenain and J. Koko "On-the-field simulation of fertilizer spreading: Part 1 Modeling," Computers and Electronics in Agriculture, Vol. 142, Part A, November 2017, pp. 235-247.

[8] J. Koko and T. Virin, "Optimization of a fertilizer spreading process," Mathematics and Computers in Simulation, Vol. 79, No. 10, June 2009, pp. 3099-3109.

[9] A. Aphale, N. Bolander, J. Park, L. Shaw, J. Svec and C. Wassgren, "Granular Fertiliser Particle Dynamics on and off a Spinner Spreader," Biosystems Engineering, Vol. 85, No. 3, July 2003, pp. 319-329.

[10] J. W. Hofstee, "Handling and Spreading of Fertilizers: Part 4, The Reciprocating Spout Type Fertilizer Spreader," Journal of Agricultural Engineering Research, Vol. 62, No. 1, September 1995, pp. 9-24.

[11] J. W. Hofstee, "Handling and Spreading of Fertilizers: Part 5, The Spinning Disc Type Fertilizer Spreader," Journal of Agricultural Engineering Research, Vol. 62, No. 3, November 1995, pp. 143-162.

[12] J. W. Hofstee, "Handling and Spreading of Fertilizers: Part 3, Measurement of Particle Velocities and Directions with Ultrasonic Transducers, Theory, Measurement System, and Experimental Arrangements," Journal of Agricultural Engineering Research, Vol. 58, No. 1, May 1994, pp. 1-16.

[13] S. Yinyan, C. Man, W. Xiaochan, M. Oluoch Odhiambo and D. Weimin, "Numerical simulation of spreading performance and distribution pattern of centrifugal variable-rate fertilizer applicator based on DEM software," Computers and Electronics in Agriculture, Vol. 144, January 2018, pp. 249-259.

[14] S. Villette, E. Piron and D. Miclet, "Hybrid centrifugal spreading model to study the fertiliser spatial distribution and its assessment using the transverse coefficient of variation," Computers and Electronics in Agriculture, Vol. 137, May 2017, pp. 115-129.

[15] S. Villette, E. Piron F. Cointault, and B. Chopinet, "Centrifugal spreading of fertiliser: Deducing three-dimensional velocities from horizontal outlet angles using computer vision,” Biosystems Engineering, Vol. 99, No. 4, April 2008, pp. 496-507.

[16] F. Ahmed Shah and M. Aslam, Noor "Some numerical methods for solving nonlinear equations by using decomposition technique," Applied Mathematics and Computation,Vol. 251, 15 January 2015, pp. 378-386.

[17] S. Nemati, P. Lima and S. Sedaghat, "An effective numerical method for solving fractional pantograph differential equations using modification of hat functions," Applied Numerical Mathematics, Vol. 131, September 2018, pp. 174-189. 